\title{
Automatic Construction and Refinement of a Class Hierarchy over Multi-valued Data
}

\author{
Nathalie Pernelle, Marie-Christine Rousset, and Véronique Ventos \\ Université Paris-Sud \\ LRI Bat. 490 \\ 91405 Orsay, France \\ \{pernelle,mcr, ventos\}@lri.fr
}

\begin{abstract}
In many applications, it becomes crucial to help users to access to a huge amount of data by clustering them in a small number of classes described at an appropriate level of abstraction. In this paper, we present an approach based on the use of two languages of description of classes for the automatic clustering of multi-valued data. The first language of classes has a high power of abstraction and guides the construction of a lattice of classes covering the whole set of the data. The second language, more expressive and more precise, is the basis for the refinement of a part of the lattice that the user wants to focus on.
\end{abstract}

\section{Introduction}

The main goal of our approach is to help users to access to a huge amount of multi-valued data by clustering them in a small number of classes organized in a hierarchy and described at an appropriate level of abstraction. The data (i.e. instances) we want to treat are described by a various set of attributes that can be multi-valued and each instance is labelled by the name of a basic type. The approach is based on the use of two languages of description of classes. The first language of classes has a high power of abstraction and guides the construction of a coarse lattice covering the whole set of the data. The second language, more expressive and more precise, is the basis for the refinement of a part of the lattice that the user wants to focus on. This second language actually allows us to distinguish new clusters. Besides, we exploit the fact that the data are labelled: we gather instances sharing the same label in basic classes and we construct clusters of basic classes rather than clusters of instances.

In Sect. 2, we present languages of instances and classes together with some basic operations used in the lattice construction steps. Section 3 describes the first clustering algorithm leading to the construction of a crude lattice. A part of this lattice may be refined using the second clustering algorithm described in Sect. 4. Our approach has been implemented and experimented on real data in the setting of the GAEL project which aims at building flexible electronic catalogs. Our experiments have been conducted on real data coming from the $\mathrm{C} /$ Net electronic catalog of computer products (http://www.cnet.com). Experimental results are presented in Sect. 5. 


\section{Languages of Instances and Classes}

In this section, we define the language of instances, $\mathcal{L}_{1}$, in which we describe multi-valued data, and the two languages of classes $\mathcal{L}_{2}$ and $\mathcal{L}_{3}$ that we use to describe classes over those data, at two levels of abstraction. First, we provide some notations and preliminaries.

\subsection{Preliminaries and Notations}

Given a language of instances, a language of classes $\mathcal{L}$ defines the expressions that are allowed as class descriptions. A class description is intended to represent in an abstract and concise way the properties that are common to the set of its instances. A membership relation, denoted by $i s a_{\mathcal{L}}$, establishes the necessary connection between a given language of instances and an associated language of classes $\mathcal{L}$.

Definition 1 (Extension of a class description). Let $I$ be a set of instances, and $C$ a $\mathcal{L}$ class description. The extension of $C$ w.r.t $I$ is the following set:

$$
\operatorname{ext}_{I}(C)=\left\{i \in I \mid \text { iisa }_{\mathcal{L}} C\right\}
$$

The subsumption relation is a preorder relation between class descriptions, induced by the inclusion relation between class extensions.

Definition 2 (Subsumption between classes). Let $C_{1}$ and $C_{2}$ be two $\mathcal{L}$ class descriptions. $C_{1}$ is subsumed by $C_{2}$, denoted $C_{1} \preceq_{\mathcal{L}} C_{2}$, iff for every set I of instances, $\operatorname{ext}_{I}\left(C_{1}\right) \subseteq \operatorname{ext}_{I}\left(C_{2}\right)$.

In Sects. 2.3 and 2.4 we will provide a constructive characterization of subsumption for the two languages of classes that we consider.

The notion of abstraction of an instance in a language of classes $\mathcal{L}$ corresponds, when it exists, to the most specific class description in $\mathcal{L}$ which it is an instance of.

Definition 3 (Abstraction of an instance). Let $i$ be an instance, the $\mathcal{L}$ class description $C$ is an abstraction of $i$ in $\mathcal{L}$ (for short $C=a b s_{\mathcal{L}}(i)$ ) iff

1. $i$ isa $a_{\mathcal{L}} C$, and

2. if $D$ is a class description such that $i$ isa $a_{\mathcal{L}} D$, then $C \preceq{ }_{\mathcal{L}} D$.

The notion of least common subsumer will be the basis for gathering classes in our clustering algorithm.

\section{Definition 4 (Least Common Subsumer).}

Let $C_{1}, \ldots, C_{n}$ be class descriptions in $\mathcal{L}$. The $\mathcal{L}$ class description $C$ is a least common subsumer of $C_{1}, \ldots, C_{n}$ in $\mathcal{L}$ (for short $C=l \operatorname{cs} \mathcal{L}\left(C_{1}, \ldots, C_{n}\right)$ ) iff

1. $C_{i} \preceq_{\mathcal{L}} C$ for all $1 \leq i \leq n$, and

2. if $D$ is a class description satisfying $C_{i} \preceq_{\mathcal{L}} D$ for all $1 \leq i \leq n$, then $C \preceq \mathcal{L} D$ 


\subsection{The Language of Instances}

The data that serve as instances of the classes that we build are typed (i.e., labelled by the name of a basic type) and described by a set of pairs (Attribute, Value). The attributes used for describing the data may vary from an item to another. In addition, an attribute can be multi-valued.

Definition 5 (Terms of $\mathcal{L}_{1}$ ). Let $\mathcal{B}$ be a finite set of basic types, $\mathcal{A}$ a finite set of attributes, and $\mathcal{V}$ a set of values. A term of $\mathcal{L}_{1}$ is of the form:

$$
\left\{c, a t t_{1}=V_{1}, \ldots, a t t_{n}=V_{n}\right\}
$$

where $c \in \mathcal{B}, \forall i \in[1 . . n]$, att $_{i} \in \mathcal{A}$ and $V_{i} \subseteq \mathcal{V}$.

The description of an instance is a term of $\mathcal{L}_{1}$. For example, we can find a product in the $\mathrm{C} / \mathrm{Net}$ catalog, whose $\mathcal{L}_{1}$ description is:

$\{$ RemovableDiskDrive, $C D / D V D / T y p e=\{C D R W\}$,

StorageRemovableType $=\{$ SuperDisk $\}$, Compatibility $=\{M A C, P C\}\}$

In the following, we will consider that the type $c$ of a $\mathcal{L}_{1}$ description is a boolean attribute.

\subsection{The Language of Classes $\mathcal{L}_{2}$}

Definition 6 (Class description in $\mathcal{L}_{2}$ ). $A \mathcal{L}_{2}$ class description (of size $n$ ) is a tuple of attributes $\left\{a_{1} t_{1}, \ldots, a_{t}\right\}$, where $\forall i \in[1 . . n]$, att $t_{i} \in \mathcal{A}$.

The connection between the language of instances $\mathcal{L}_{1}$ and the language of classes $\mathcal{L}_{2}$ is based on the following definition of the membership relation.

Definition 7 (Membership relation for $\mathcal{L}_{2}$ ). Let $i$ be an instance description in $\mathcal{L}_{1}$. Let $C$ be a $\mathcal{L}_{2}$ class description: $i$ is an instance of $C$ iff every attribute appearing in $C$ also appears in $i$.

The following proposition, whose proof is straightforward, characterizes subsumption, least common subsumer and abstraction in $\mathcal{L}_{2}$.

\section{Proposition 1 (Properties of $\mathcal{L}_{2}$ ).}

- Let $C_{1}$ and $C_{2}$ be two $\mathcal{L}_{2}$ class descriptions. $C_{1} \preceq_{\mathcal{L}_{2}} C_{2}$ iff every attribute of $C_{2}$ is also an attribute of $C_{1}$.

- Let $\left\{a_{1} t_{1}=V_{1}, \ldots\right.$, att $\left.{ }_{n}=V_{n}\right\}$ be an instance description in $\mathcal{L}_{1}$. Its abstraction in $\mathcal{L}_{2}$ is unique: it is $\left\{a t t_{1}, \ldots, a t t_{n}\right\}$.

- Let $C_{1}, \ldots, C_{n}$ be $n \mathcal{L}_{2}$ class descriptions. Their least common subsumer is unique: it is made of the set of attributes that are common to all the $C_{i}$ 's. 


\subsection{The Language of Classes $\mathcal{L}_{3}$}

$\mathcal{L}_{3}$ is richer than $\mathcal{L}_{2}$ on different aspects: it makes possible to restrict the possible values of an attribute ; it enables to distinguish the number of values of an attribute through different suffixes $(*,+, ?, \epsilon)$ whose notation is inspired by the one used in XML for describing document type definitions (DTDs), and whose formal semantics corresponds to standard description logics constructors.

Definition 8 (Class description in $\mathcal{L}_{3}$ ). $A \mathcal{L}_{3}$ class description (of size $n$ ) is a tuple

$$
\left\{a t t_{1}^{s u f f_{1}}: V_{1}, \ldots, a t t_{n}^{s u f f_{n}}: V n\right\}
$$

where $\forall i \in[1 . . n]$, att $_{i} \in \mathcal{A}, V_{i} \subseteq \mathcal{V}$, and suff $f_{i} \in\{*,+, ?, \epsilon\}$

The following definition formalizes the membership relation between an instance and a class description in $\mathcal{L}_{3}$.

Definition 9 (Membership relation for $\mathcal{L}_{3}$ ). Let $i$ be an instance description in $\mathcal{L}_{1}$. Let $C$ be a $\mathcal{L}_{3}$ class description. $i$ is an instance of $C$ iff every attribute in $i$ appears in $C$ and for every term att ${ }^{\text {suff }}: V$ appearing in $C$,

- when suff $f=*$, if there exists $V^{\prime}$ s.t att $=V^{\prime} \in i$, then $V^{\prime} \subseteq V$,

- when suff $=+$, there exists $V^{\prime} \subseteq V$ s.t att $=V^{\prime} \in i$,

- when suff $f=$ ?, if there exists $V^{\prime}$ s.t att $=V^{\prime} \in i$, then $V^{\prime}$ is a singleton and $V^{\prime} \subseteq V$

- when suff $f=\epsilon$, there exists $V^{\prime}$ singleton s.t $V^{\prime} \subseteq V$ and att $=V^{\prime} \in i$.

The product described in 2.2 is an instance of the $\mathcal{L}_{3}$ class description $C_{1}$ :

$\left\{\right.$ RemovableDiskDrive $^{\epsilon}:\{$ true,$C D / D V D /$ ReadSpeed $?:\{20 x, 32 x, 24 x\}$, $C D / D V D /$ Type $^{\epsilon}:\{C D R O M, C D R W\}$, Compatibility ${ }^{+}:\{M A C, P C\}$,

StorageRemovableType $\epsilon^{\epsilon}\{$ SuperDisk, ZIP, JAZ\}\}

It represents the set of products that have in their description (i) necessarily the monovalued and boolean attribute RemovableDiskDrive whose value must be true, (ii) possibly the attribute $C D / D V D /$ ReadSpeed, and if that is the case, this attribute is monovalued and its value belongs to the set $\{20 x, 32 x, 24 x\}$, (iii) necessarily the attribute $C D / D V D /$ Type, which is monovalued and takes its value in the set $\{C D R O M, C D R W\}$, (iv) necessarily the attribute Compatibility, which can be multivalued and takes its value(s) in the set $\{M A C, P C\}$, (v) necessarily the attribute StorageRemovableType, which is monovalued and takes its value in the set $\{$ SuperDisk, $Z I P, J A Z\}$.

The following propositions state the main properties of $\mathcal{L}_{3}$. Their proofs follow from results in tractable description logics where structural subsumption is complete.

Proposition 2 (Characterization of subsumption in $\mathcal{L}_{3}$ ). Let $C_{1}$ and $C_{2}$ be two $\mathcal{L}_{3}$ class descriptions. $C_{1} \preceq_{\mathcal{L}_{3}} C_{2}$ iff all the attributes appearing in $C_{1}$ appear also in $C_{2}$ and for every pair att ${ }^{\text {suff }}: V$ appearing in $C_{2}$, - when suff $f=*$, if there exists att ${ }^{\text {suff }} f^{\prime}: V^{\prime} \in C_{1}$, then $V^{\prime} \subseteq V$, 
- when suf $f=+$, there exists $V^{\prime} \subseteq V$ s.t att ${ }^{+}: V^{\prime} \in C_{1}$ or att ${ }^{\epsilon}: V^{\prime} \in C_{1}$

- when suff $=$ ?, if there exists att ${ }^{\text {suff } f^{\prime}}: V^{\prime} \in C_{1}$, then suff $f^{\prime}=$ ? or suff $f^{\prime}=\epsilon$, and $V^{\prime} \subseteq V$,

- when suff $f=\epsilon$, there exists $V^{\prime}$ s.t $V^{\prime} \subseteq V$ and att ${ }^{\epsilon} V^{\prime} \in C_{1}$.

Proposition 3 (Characterization of abstraction in $\mathcal{L}_{3}$ ). Let $\left\{a t t_{1}=V_{1}, \ldots\right.$, att $\left._{n}=V_{n}\right\}$ be an instance description in $\mathcal{L}_{1}$. Its abstraction in $\mathcal{L}_{3}$ is unique: $a b s_{\mathcal{L}_{3}}=\left\{a t t_{1}^{s u f f_{1}}: V_{1}, \ldots\right.$, att $\left._{n}^{\text {suff }} f_{n}: V_{n}\right\}$, where $\forall i \in[1 . . n]$, if $\left|V_{i}\right| \geq 2$ then suf $f_{i}=+$ else suff $f_{i}=\epsilon$.

Proposition 4 (Characterization of lcs in $\mathcal{L}_{3}$ ). Let $C_{1}, \ldots, C_{n}$ be $n \mathcal{L}_{3}$ class descriptions. Let $A$ be the set of attributes belonging to at least one description $C_{i} . C_{1}, \ldots, C_{n}$ have a unique least common subsumer in $\mathcal{L}_{3}$, whose description is characterized as follows:

- for every attribute att $\in A$, let $V$ be the union of the sets of values associated with att in the class descriptions $C_{i}$ 's: $V=\bigcup_{1}^{n}\left\{v \in V_{i} \mid\right.$ att $\left.^{\text {suff }}: V_{i} \in C_{i}\right\}$.

- att $^{\epsilon}: V \in \operatorname{lcs}\left(C_{1}, \ldots, C_{n}\right)$ iff att ${ }^{\epsilon}: V_{i} \in C_{i} \forall i \in[1 . . n]$.

- att $?: V \in \operatorname{lcs}\left(C_{1}, \ldots, C_{n}\right)$ iff

$\left(\forall i \in[1 . . n]\right.$ att ${ }^{*}: V_{i} \notin C_{i}$ and $\left.a t t^{+}: V_{i} \notin C_{i}\right)$, and

- either $\exists i \in[1 . . n]$ s.t. $a t t^{\text {? }}: V_{i} \in C_{i}$,

- or $\exists i \in[1 . . n]$ s.t. att ${ }^{s} V^{\prime} \notin C_{i}$ for any $s$.

- att* $: V \in l c s\left(C_{1}, \ldots, C_{n}\right)$ iff

- either $\exists i \in[1 . . n]$ s.t. att ${ }^{*}: V_{i} \in C_{i}$,

- or $\exists i \in[1 . . n]$ s.t. att ${ }^{+}: V_{i} \in C_{i}$, and $\exists j \in[1 . . n]$ s.t. att ${ }^{\text {? }}: V_{j} \in C_{j}$ or att $s^{s^{\prime}}: V^{\prime} \notin C_{j}$ for any suffix $s^{\prime}$.

- $a t t^{+}: V \in \operatorname{lcs}\left(C_{1}, \ldots, C_{n}\right)$ iff

$\exists i \in[1 . . n]$ s.t. att ${ }^{+}: V_{i} \in C_{i}$ and $\forall j \in[1 . . n]$, att ${ }^{+}: V_{j} \in C_{j}$ or att ${ }^{\epsilon} V_{j} \in C_{j}$

For example, if $C_{2}$ is the $\mathcal{L}_{3}$ description:

$\left\{\right.$ Compatibility $^{*}:\{P C$, Unix $\}$, StorageRemovableType $e^{\epsilon}:\{D A T\}$,

CompressedCapacity $\left.{ }^{\epsilon}:\{8,24,32,70\}\right\}$

$\operatorname{lcs}\left(C_{1}, C_{2}\right)=$

$\{$ RemovableDiskDrive? : $\{$ true $\}, C D / D V D /$ ReadSpeed $:\{20 x, 32 x, 24 x\}$,

$C D / D V D / T y p e^{?}:\{C D R O M, C D R W\}, C o m p a t i b i l i t y *:\{M A C, P C, U n i x\}$,

StorageRemovableType ${ }^{\epsilon}:\{$ SuperDisk, ZIP, JAZ,DAT\},

CompressedCapacity? $:\{8,24,32,70\}\}$

\section{Construction of a Lattice of $\mathcal{L}_{2}$ Classes}

The goal is to structure a set of data described in $\mathcal{L}_{1}$ into clusters labelled by $\mathcal{L}_{2}$ descriptions, and organized in a lattice providing a browsable semantic interface facilitating the access to the data for end-users. We proceed in two steps: 
1. In the first step, the data are partitioned according to their type: for each type $c$, we create a basic class named $c$. Its set of instances, denoted inst $(c)$, is the set of data of type $c$. Its $\mathcal{L}_{2}$ description, $\operatorname{desc}(c)$, is obtained by computing the least common subsumer of the abstractions of its instances. The result of this step is a set $\mathcal{C}$ of basic classes and a set $\mathcal{A}$ of attributes supporting the $\mathcal{L}_{2}$ descriptions of the classes of $\mathcal{C}$. For each attribute $a$, the set classes $(a)$ of basic classes having $a$ in their description is computed. This preliminary clustering step has a linear data complexity.

2. In the second step, a lattice of clusters is constructed by gathering basic classes according to similarities of their $\mathcal{L}_{2}$ descriptions. In this step, clusters are unions of basic classes. The computational complexity of this step does not depend on the number of initial data but only on the size of the $\mathcal{L}_{2}$ descriptions of basic classes.

We now detail this second step. A cluster $c_{i_{1}} \ldots c_{i_{k}}$ will appear in the lattice if the $\mathcal{L}_{2}$ descriptions of the classes $c_{i_{1}} \ldots c_{i_{k}}$ are judged similar enough to gather their instances. The similarity between class descriptions is stated by attributes in common. However, we take into account only attributes that do not occur in too many classes. For instance, the attribute price may appear in all the instances of a catalog describing products, and is therefore not useful to discriminate product descriptions. Among the set $\mathcal{A}$ of attributes, we select meaningful attributes as being the attributes $a \in \mathcal{A}$ such that $\frac{|\operatorname{classes}(a)|}{|\operatorname{classes}|} \leq s$ where $s$ is a certain threshold (e.g., $s=0.8$ ). Let $\mathcal{A}_{0}$ be the set of meaningful attributes. We redescribe all the basic classes in terms of the attributes of $\mathcal{A}_{0}$ only: for a basic class $c$, we call its short description, denoted shortdesc(c), the $\mathcal{L}_{2}$ description of $c$ restricted to the meaningful attributes: $\operatorname{shortdesc}(c)=\operatorname{desc}(c) \cap \mathcal{A}_{0}$.

Our clustering algorithm, $\mathcal{L}_{2}$-Cluster, is described in Algorithm 1 . It is adapted from a frequent item set algorithm ([2]). It iteratively builds levels of clusters, starting with building the level of the coarsest clusters corresponding to unions of basic classes having atleast one attribute in common. Each iteration $k$ is guided by attribute sets of increasing size $k$ which, being common to some class descriptions, are the support of the creation of a potential node gathering those classes. Among those potential nodes, we effectively add to the lattice those whose $\mathcal{L}_{2}$ short description is equal to their $k$-support: the $k$-support of a node generated at iteration $k$ is the $k$-itemset supporting the generation of that node. By doing so, we guarantee that the description of the nodes added to the lattice is strictly subsumed by those of their fathers.

Notation: We call a $k$-itemset a set of attributes of size $k$. We assume that attributes in itemsets are kept sorted in their lexicographic order. We use the notation $p[i]$ to represent the $i$-th attribute of the $k$-itemset $p$ consisting of the attributes $p[1], \ldots, p[k]$ where $p[1]<\ldots<p[k]$.

Figure [1] shows the lattice returned by $\mathcal{L}_{2}$-Cluster when it is applied on $\mathcal{C}=$ $\left\{c_{1}, c_{2}, c_{3}, c_{4}, c_{5}\right\}$ and $\mathcal{A}=\left\{a_{1}, a_{2}, a_{3}, a_{4}\right\}$ such that: $\operatorname{shortdesc}\left(c_{1}\right)=\left\{a_{1}, a_{2}, a_{3}\right\}$ $\operatorname{shortdesc}\left(c_{2}\right)=\left\{a_{2}\right\} \quad \operatorname{shortdesc}\left(c_{3}\right)=\left\{a_{1}, a_{3}\right\} \operatorname{shortdesc}\left(c_{4}\right)=\left\{a_{3}, a_{4}\right\}$ $\operatorname{shortdesc}\left(c_{5}\right)=\left\{a_{1}, a_{3}\right\}$ 


\section{Algorithm 1. $\mathcal{L}_{2}$-Cluster}

Require: a set $\mathcal{A}_{0}$ of meaningful attributes: for each $a \in \mathcal{A}_{0}, \operatorname{classes}(a)$ is the set of basic classes of $\mathcal{C}$ whose $\mathcal{L}_{2}$ short description contains $a$.

Ensure: return a lattice organized in levels of nodes. Each node $n$ is characterized by classes $(n)$ : the basic classes it gathers, and $\operatorname{shortdesc}(n)$ : the least common subsumer of the short description of the basic classes of the cluster.

1: (* Initialization step gathering the biggest unions of classes having at least one attribute in common:*)

2: $\mathcal{A}_{1} \leftarrow \mathcal{A}_{0}$, level $(1) \leftarrow \emptyset, \ldots$, level $(|\mathcal{C}|) \leftarrow \emptyset$

3: for every $a \in \mathcal{A}_{1}$ do

4: $\quad$ let $\operatorname{classes}(a)=\left\{c_{1}^{a}, \ldots, c_{j}^{a}\right\}$

5: $\quad$ let $\operatorname{desc}=\operatorname{lcs}_{\mathcal{L}_{2}}\left(\operatorname{desc}\left(c_{1}^{a}\right), \ldots, \operatorname{desc}\left(c_{j}^{a}\right)\right)$

6: $\quad$ if $\operatorname{desc} \cap \mathcal{A}_{0}=\{a\}$ then

7: $\quad$ add to level $(j)$ a node $n$ such that: $\operatorname{classes}(n)=\left\{c_{1}^{a}, \ldots, c_{j}^{a}\right\}$; $\operatorname{shortdesc}(n)=\operatorname{desc} \cap \mathcal{A}_{0}$; $\operatorname{node}(\{a\})=n$

8: $k \leftarrow 1$

9: (* Generation of new nodes supported by $k+1$-itemsets : $\left.{ }^{*}\right)$

10: repeat

11: $\quad$ for every pair $(p, q) \in \mathcal{A}_{k}$ do

12: $\quad$ if $p[1]=q[1], \ldots, p[k-1]=q[k-1], p[k]<q[k]$ then

13: $\quad$ let $n e w p=p \cup\{q[k]\}$, and let $\mathcal{S}_{k}$ be the set of $k$-subsets of newp.

14: $\quad$ if $\mathcal{S}_{k} \subseteq \mathcal{A}_{k}$ and classes $(\operatorname{node}(p)) \cap \operatorname{classes}(q[k]) \neq \emptyset$ then

15: $\quad$ add newp to $\mathcal{A}_{k+1}$

16: $\quad$ let $\left\{c_{i_{1}}, \ldots, c_{i_{j}}\right\}$ be classes $(\operatorname{node}(p)) \cap \operatorname{classes}(q[k])$

17: $\quad$ let $\operatorname{desc}=\operatorname{lcs}{\mathcal{\mathcal { L } _ { 2 }}}_{2}\left(\operatorname{desc}\left(c_{i_{1}}\right), \ldots, \operatorname{desc}\left(c_{i_{j}}\right)\right)$

18: $\quad$ if desc $=$ newp then

19: $\quad$ add to $\operatorname{level}(j)$ a node $n$ such that: classes $(n)=\left\{c_{i_{1}}, \ldots, c_{i_{j}}\right\}$; $\operatorname{shortdesc}(n)=\operatorname{desc}$;

20: $\quad k \leftarrow k+1$

$$
\operatorname{node}(\text { newp })=n
$$

21: until $\mathcal{A}_{k}=\emptyset$

22: (* Creation of the lattice. For every node $n$, Fathers $(n)$ group the fathers of $n$ among the nodes of greater levels:*)

23: Initialize Fathers $(n)$ and AncNotFathers $(n)$ to $\emptyset$ for every generated node $n$.

24: for $i=|\mathcal{C}|-1$ downto 1 do

25: for every node $n \in \operatorname{level}(i)$ do

26: $\quad$ for $j=i+1$ to $|\mathcal{C}|$ do

27:

$28:$

for every node $m \in \operatorname{level}(j)$ do

if classes $(n) \subset$ classes $(m)$ and $m \notin$ AncNotFathers $(n)$ then add $m$ to Fathers $(n)$

29: add Fathers $(m) \cup$ AncNotFathers $(m)$ to AncNotFathers $(n)$ 


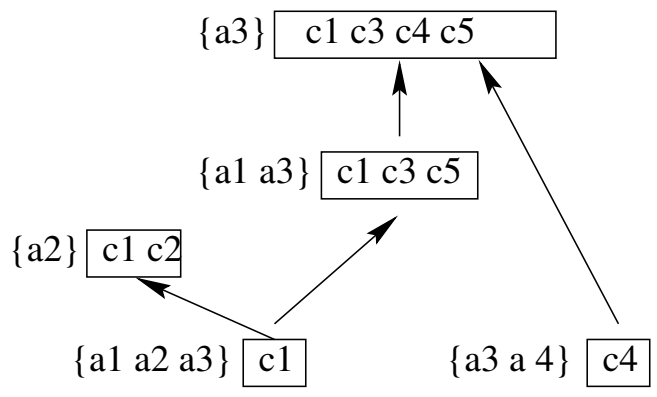

Fig. 1. Example of a lattice constructed by $\mathcal{L}_{2}$-Cluster

The following proposition summarizes the properties of the algorithm $\mathcal{L}_{2}$-Cluster.

Proposition 5 (Properties of $\mathcal{L}_{2}$-Cluster). Let $\mathcal{H}$ be the lattice returned by $\mathcal{L}_{2}$-Cluster.

- For each node $n \in \mathcal{H}$, let shortdesc $(n)$ and classes $(n)$ be respectively the description and the set of basic classes returned by $\mathcal{L}_{2}$-Cluster:

$$
\operatorname{shortdesc}(n)=\operatorname{lcs}_{\mathcal{L}_{2}}\left(\operatorname{shortdesc}\left(\operatorname{abst} t_{\mathcal{L}_{2}}\left(i_{1}\right)\right), \ldots, \operatorname{shortdesc}\left(\operatorname{abst}_{\mathcal{L}_{2}}\left(i_{k}\right)\right)\right)
$$

where $\left\{i_{1}, \ldots, i_{k}\right\}=\bigcup_{c \in \text { classes(n) }} \operatorname{inst}(c)$.

- $\mathcal{H}$ is a Galois lattice, i.e. for every node $n$, the pair (classes $(n)$, shortdesc $(n))$ is maximal in the following sense: there is no $m \in \mathcal{H}$ such that classes $(n) \subset \operatorname{classes}(m)$ and $\operatorname{shortdesc}(n)=\operatorname{shortdesc}(m)$, or $\operatorname{shortdesc}(n)$ $\prec \operatorname{shortdesc}(m)$ and classes $(n)=\operatorname{classes}(m)$.

\section{Refinement in $\mathcal{L}_{3}$}

The goal of this step is to refine a part of the lattice $\mathcal{H}$ computed by $\mathcal{L}_{2}$-Cluster based on the more expressive language $\mathcal{L}_{3}$. This step is achieved after a user chooses one node Fatn and one of its descendants Sonn in $\mathcal{H}$. Algorithm 2 describes how new nodes are possibly added between Sonn and Fatn. Those new nodes correspond to clusters whose descriptions in $\mathcal{L}_{2}$ did not distinguish from those of Fatn or Sonn, while having distinct descriptions in $\mathcal{L}_{3}$. A closure operation on those nodes is necessary in order to make their $\mathcal{L}_{3}$ descriptions maximal w.r.t the union of basic classes which they gather. $\mathcal{L}_{3}$-Cluster applies after the descriptions in $\mathcal{L}_{3}$ (denoted desc3 in Algorithm 2) have been computed for Sonn and Fatn. Those computations are least common subsumer calculations whose overall time cost is polynomial w.r.t to the size and the number of the instances of the basic classes involved in Fatn.

Let us illustrate the application of $\mathcal{L}_{3}$-Cluster on the nodes $c 1 c 3 c 5$ and $c 1$ of the lattice of Fig. 1 assuming that the $\mathcal{L}_{3}$ descriptions of the involved basic classes are: 


\section{Algorithm 2. $\mathcal{L}_{3}$-Cluster}

Require: Two nodes Fatn and Sonn such that classes $($ Sonn $) \subset \operatorname{classes}($ Fatn).

Ensure: return a lattice between Fatn and Sonn

1: L-Cl $\leftarrow\{$ classes $($ Fatn $), \operatorname{classes}($ Sonn $)\}$

2: LRes-Cl $\leftarrow$ L-Cl ; Nodes $\leftarrow\{$ Sonn $\}$

3: for every node $\mathrm{n} \in$ Nodes do

4: for every class $c \in \operatorname{classes}($ Fatn $) \backslash$ classes $(n)$ do

5: $\quad$ Change $\leftarrow$ false $;$ classes $\leftarrow$ classes $(n) \cup\{c\}$

6: $\quad$ if classes $\notin \mathrm{L}-\mathrm{Cl}$ then

7: $\quad \mathrm{L}-\mathrm{Cl} \leftarrow \mathrm{L}-\mathrm{Cl} \cup\{$ classes $\}$

8: $\quad \operatorname{desc} 3 \leftarrow \operatorname{lcs}_{\mathcal{L}_{3}}(\operatorname{desc} 3(n), \operatorname{desc} 3(c))$

9: $\quad$ (* Closure operation: *)

10: $\quad$ for every class $C l \in$ classes(Fatn) $\backslash$ classes do

11: $\quad$ if $\operatorname{desc} 3(\mathrm{Cl}) \preceq \mathcal{L}_{3} \operatorname{desc} 3$ then

12:

13 :

14 : add $C l$ to classes ; Change $\leftarrow$ true

if classes $\notin$ LRes-Cl then add a node $p$ to Nodes such that classes $(p)=$ classes and $\operatorname{desc} 3(p)=\operatorname{desc} 3$

15: $\quad$ LRes- $\mathrm{Cl} \leftarrow$ LRes-Cl $\cup\{$ classes $(p)\}$

16: $\quad$ if Change $=$ true then

17: $\quad \mathrm{L}-\mathrm{Cl} \leftarrow \mathrm{L}-\mathrm{Cl} \cup \operatorname{classes}(p)$

18: $\quad$ Suppress $n$ from Nodes

$\operatorname{desc}\left(c_{1}\right)=\left\{a t t_{1}^{+}:\{v 1, v 3\}, \operatorname{att}_{2}^{+}:\{v 2, v 4\}, \operatorname{att}_{3}^{\epsilon}:\{v 6\}\right\} \operatorname{desc}\left(c_{3}\right)=\left\{a t t_{1}^{\epsilon}:\{v 3\}\right.$, $\left.a t t_{2}^{?}:\{v 4\}, a t t_{3}^{\epsilon}:\{v 7\}\right\} \operatorname{desc}\left(c_{5}\right)=\left\{a t t_{1}^{\epsilon}:\{v 5\}, a t t_{3}^{\epsilon}:\{v 7, v 8\}\right\}$.

LRes-Cl and L-Cl are initialized to $\{\{c 1, c 3, c 5\},\{c 1\}\}$.

- Gathering $c 3$ with $c 1$ is considered first:

$\operatorname{desc} 3=\left\{a t t_{1}^{+}:\{v 1, v 3\}, a t t_{2}^{*}:\{v 2, v 4\}, a t t_{3}^{\epsilon}:\{v 6, v 7\}\right\}$, classes $=\{c 1, c 3\}$

Since $\operatorname{desc} 3(c 5)$ is not subsumed by desc3, the new node $c 1 c 3$ is added.

LRes-Cl is updated to $\{\{\mathrm{c} 1, \mathrm{c} 3, \mathrm{c} 5\},\{\mathrm{c} 1\},\{\mathrm{c} 1, \mathrm{c} 3\}\}$.

- Gathering $c 5$ with $c 1$ is now considered:

$\operatorname{desc} 3=\left\{a t t_{1}^{+}:\{v 1, v 3, v 5\}, a t t_{2}^{*}:\{v 2, v 4\}, a t t_{3}^{\epsilon}:\{v 6, v 7, v 8\}\right\}$, classes $=\{c 1, c 5\}$

Since $\operatorname{desc} 3(c 3)$ is subsumed by $\operatorname{desc} 3, c 3$ is added to classes, which is updated to $\{c 1, c 3, c 5\}$. The node corresponding to $c 1 c 5$ is not added since it is not closed, the node corrresponding to its closure $c 1 c 3 c 5$ is not added either because $\{c 1, c 3, c 5\}$ is already in LRes-Cl.

The following proposition summarizes the main properties of $\mathcal{L}_{3}$-Cluster.

Proposition 6 (Properties of $\mathcal{L}_{3}$-Cluster). Let $\mathcal{C}_{1}$ be the set of basic classes of the father node. Let $\mathcal{C}_{2}\left(\mathcal{C}_{2} \subset \mathcal{C}_{1}\right)$ be the set of basic classes of the son node. Let $\mathcal{H}_{3}$ be the lattice returned by $\mathcal{L}_{3}$-Cluster.

- For each node $n \in \mathcal{H}_{3}$, let desc3(n) and classes $(n)$ be respectively the description and the set of basic classes returned by $\mathcal{L}_{3}$-Cluster:

$$
\operatorname{desc} 3(n)=\operatorname{lcs}_{\mathcal{L}_{3}}\left(\operatorname{abst}_{\mathcal{L}_{3}}\left(i_{1}\right), \ldots, \operatorname{abst}_{\mathcal{L}_{3}}\left(i_{k}\right)\right)
$$

where $\left\{i_{1}, \ldots, i_{k}\right\}=\bigcup_{c \in \text { classes }(n)} \operatorname{inst}(c)$. 
- $\mathcal{H}_{3}$ is a complete Galois lattice, i.e. for every node n, the pair (classes $(n)$, desc $3(n))$ is maximal, and $\mathcal{H}_{3}$ contains every node verifying the maximality criteria and whose set of classes includes $\mathcal{C}_{2}$ and are included in $\mathcal{C}_{1}$.

\section{Complexity and Experimental Results}

The following results come directly from known results in description logic and in Galois lattice. Since $\mathcal{L}_{3}$ is a subset of the C-CLASSIC description logic [7, the complexity of checking subsumption in $\mathcal{L}_{3}$ is quadratic w.r.t the maximal size of class descriptions and computing the lcs of $\mathcal{L}_{3}$ descriptions is linear in the number of descriptions and quadratic in their size. The worst time complexity of $\mathcal{L}_{2}$-Cluster is exponential in the maximal size of the basic classes $\mathcal{L}_{2}$ descriptions. The worst time complexity of $\mathcal{L}_{3}$-Cluster is exponential w.r.t | $\operatorname{classes}($ Fatn $) \mid$ $-\mid \operatorname{classes}($ Sonn) $\mid$.

We have evaluated our approach using a real dataset composed of 2274 computer products extracted from the $\mathrm{C} / \mathrm{Net}$ catalog. Each product is described using a subset of 234 attributes, possibly multi-valued. There are 59 types of products and each product is labelled by one and only one type. The goal of the experiment was twofold: to assess the efficiency and the simplicity of the lattice for the first clustering step $\left(\mathcal{L}_{2}\right.$-Cluster $)$ and to show the accuracy of the refinement of a part of the lattice using the second clustering step $\left(\mathcal{L}_{3}\right.$-Cluster $)$.

In order to make the $\mathcal{L}_{2}$ lattice even simpler, the number of nodes obtained with $\mathcal{L}_{2}$-Cluster may be parametrized by a threshold $\mathrm{n}$ used to restrict the nodes that appear in the lattice to gather at least $\mathrm{n}$ basic classes (13]). Figure 2 shows that, as it is mentionned in 15, this quantitative criteria allows us to significantly decrease the size of the lattice. Figure 3 illustrates the simplicity of the $\mathcal{L}_{2}$ descriptions and the significance of the nodes.

$\mathcal{L}_{3}$-Cluster allows to distinguish nodes that cannot be distinguished by $\mathcal{L}_{2}$ Cluster. For instance, if $\mathcal{L}_{3}$-Cluster is applied when an end-user chooses to refine the $\mathcal{L}_{2}$ lattice between the node (a) and the node (b) in Fig. 3, the aggregation of all types of drivers (i.e. RemovableTapeDrive, RemovableDiskDrive and HardDiskDrive) is part of the $\mathcal{L}_{3}$ lattice. This new cluster appears for the following reasons: no driver is described using the attributes Stor.Controller/ RAIDLevel, Networking/DataLinkProtocol or Networking/Type (those at-

\begin{tabular}{|l|c|c|c|c|c|}
\hline Threshold & 1 & 2 & 3 & 4 & 5 \\
\hline Number of nodes & 119 & 60 & 24 & 13 & 9 \\
\hline Maximal depth & 4 & 3 & 2 & 1 & 1 \\
\hline Running Times (s) & 12.3 & 10.4 & 2.1 & 1.1 & 1 \\
\hline
\end{tabular}

Fig. 2. Quantitative results of $\mathcal{L}_{2}$-Cluster 


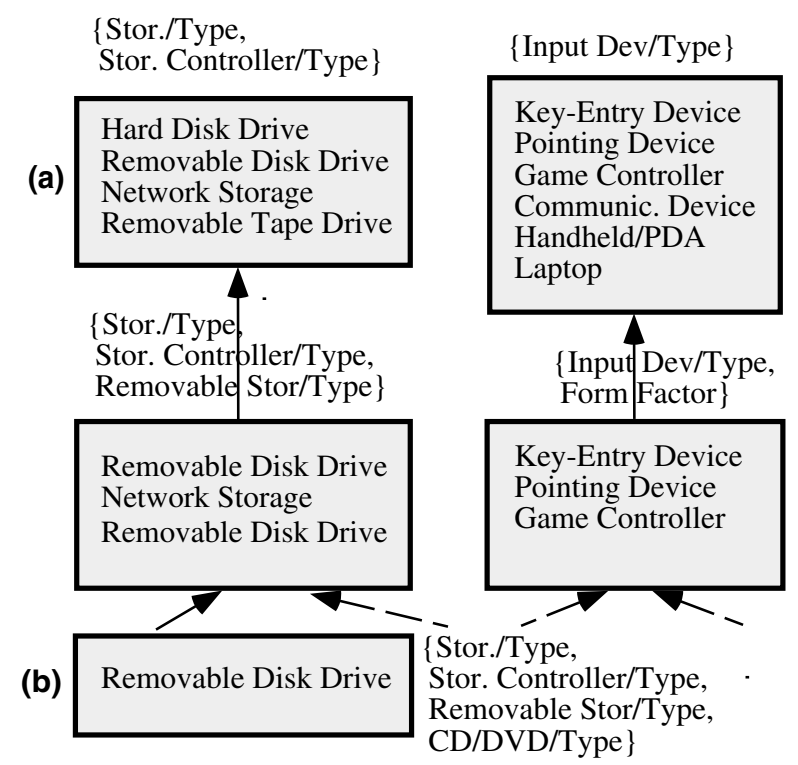

Fig. 3. A part of the $\mathcal{L}_{2}$ lattice for $\mathrm{C} /$ net $(\mathrm{n}=3)$

tributes were optional in $\mathcal{L}_{3}$ description of (a)). In addition, the value $S C S I$ for the attribute Stor.Controller/Type is not a possible value for a driver.

\section{Conclusion and Discussion}

This paper has proposed an approach to organize into clusters large sets of semistructured data. The scaling up of the approach is made possible because its complexity is remained in control in different ways: (1) the data are aggregated into basic classes and the clustering applies on the set of those basic classes instead of applying on the data set (2) the two-step clustering method first builds a coarse hierarchy, based on a simple language for describing the clusters, and uses a more elaborate language for refining a small subpart of the hierarchy delimited by two nodes.

Related Work: Our work can be compared with existing work in machine learning based on more expressive languages than propositional language and/or using a shift of representation. Most work on expressive languages has been developped in a supervised setting (e.g. Inductive Logic Programming), while little work exists in an unsupervised setting. We can cite KBG 4, TIC [5] and [11] which perform clustering in a relational setting. The main difference with our approach is that they use a distance as a numerical estimation of similarity. Although the best representation of a cluster is the least common subsumer of its instances, they approximate it numerically by the cluster centroid (i.e., 
the point that minimizes the sum of squared distances). The reason is that, in contrast with our setting where the lcs computation in $\mathcal{L}_{3}$ is polynomial, lcs computing in their first-order language may be exponential. KLUSTER [10] refines a basic taxonomy of concepts in the setting of a description logic for which computing lcs is polynomial. In KLUSTER, the clusters are not unions but subconcepts of primitive concepts, and the refinement aims at learning discriminating definitions of mutually disjoint subconcepts of a same concept. As for the use of a shift of representation, it is used in supervised learning in order to increase accuracy (i.e. the proportion of correctly predicted concepts in a set of test examples) [8]14] or to search efficiently a reduced space of concepts [9,6]. In unsupervised learning, shift of representational bias may be used to change the point of view about the data. For instance, Cluster/2 12 provides a user with a set of parameters about his preferences on the concepts to be created. Finally, the two-step clustering approach proposed in [1] is similar in spirit with our clustering in $\mathcal{L}_{2}$ since it first identifies basic clusters (as high density clusters) before building more general clusters that are unions of those basic clusters.

Perspectives: We plan to extend our current work to take nested attributes and textual values into account in $\mathcal{L}_{3}$ in order to fully deal with XML data. Another relevant perspective of this work is the discovery of associations [3].

\section{References}

1. R. Agrawal, J. Gehrke, D. Gunopulos, and P. Raghavan. Automatic subspace clustering of high dimensional data for data mining applications. SIGMOD Record, 27:94-105, 1998.

2. R. Agrawal and R. Srikant. Fast algorithms for mining association rules. VLDB-94.

3. F. Baader Computing a minimal representation of the subsumption lattice of all conjunctions of concepts defined in a terminology. KRUSE-95.

4. G. Bisson. Conceptual clustering in a first order logic representation. ECAI-92.

5. H. Blockeel, L. De Raedt, and J. Ramon. Top-down induction of clustering trees. ICML-98.

6. P. Brézellec and H. Soldano. Tabata: a learning algorithm performing a bidirectional search in a reduced search space using a tabu strategy. ECAI-98.

7. W. W. Cohen and H. Hirsh. Learning the CLASSIC description logic: Theoretical and experimental results. KR-94.

8. W. Van de Velde. Learning through progressive refinement. European Working Session on Learning (EWSL'98),Pitman editor.

9. J. Ganascia. Tdis: an algebraic formalization. IJCAI-93.

10. J. U. Kietz and K. Morik. A polynomial approach to the constructive induction of structural knowledge. Machine Learning, 14(2):193-217, 1994.

11. Mathias Kirsten and Stephan Wrobel. Extending k-means clustering to first-order representations. ILP 2000.

12. R.S. Michalski and R.E. Stepp. Learning from observation: Conceptual clustering. Machine learning: An artificial intelligence approach Vol 1, Morgan Kaufmann editor, 1983. 
13. N. Pasquier, Y.Bastide, R.Taouil and L. Lakhal. Efficient mining of association rules using closed itemset lattices. Information Systems (IS) Vol 24, 1999.

14. P. E. Utgoff. Shift of bias for inductive concept learning. Machine learning: An artificial intelligence approach Vol. 2, Morgan Kaufmann editor, 1986.

15. R. Wille. Restructuring lattice theory. Symposium on Ordered Sets, Boston, 1982. 